\title{
Trapping with biased diffusion species
}

\author{
Alejandro D. Sánchez * \\ Grupo de Física Estadística ${ }^{\dagger}$ \\ Centro Atómico Bariloche (CNEA) and Instituto Balseiro (UNC), \\ 8400 San Carlos de Bariloche, Argentina
}

\begin{abstract}
We analyze a trapping reaction with a single penetrable trap, in a one dimensional lattice, where both species (particles and trap) are mobile and have a drift velocity. We obtain the density as seen from a reference system attached to the trap and from the laboratory frame. In addition we study the nearest neighbor distance to the trap. We exploit a stochastic model previously developed, and compare the results with numerical simulations, resulting in an excellent agreement.
\end{abstract}

\section{INTRODUCTION}

With the aim of obtaining a description of diffusion-limited reactions several generalizations of the classical Smoluchowski model have been proposed [1]. A stochastic model (SM) coming from the theory of nuclear reactors [2, 3] has been adapted to model these reactions [4:5]. This framework results to be very adequate to describe different situations, making it possible to obtain results in excellent agreement with simulations [4] 14], particularly when the model equation can be exactly solved.

The trapping reaction $A+B \rightarrow B$ with a single trap and a uniform initial density of particles in a one-dimensional lattice is one of the simplest cases describing a certain kind of chemical reaction. In the present work we analyze this situation within the framework of the SM, when both species are mobile and have a drift.

Diffusion-controlled reactions in presence of a biasing field are of great interest in many areas. Let us mention a few instances: (a) Due to the gravity field, all diffusion processes that take place over the earth are affected in some quantity. For example, gravity is responsible for the drift of water molecules diffusing underground [15, 16. (b)In positron tomography [17] the addition of a strong field perpendicular to the surface leads to greater sample penetration. (c) In problems of electromigration [18] the model discussed here could be used to analyze the effect of a trapping centers on carrier concentration.

A situation related to the presented here was studied in Ref. [19], where the case of a stationary and impenetrable trap was analyzed. In the mentioned work a diffusion equation with adequate boundary conditions is the base of calculations. We want to remark that in this paper we model a standard trap, where the particles can eventually pass from left to right of it (or vice versa) without being trapped. As we will see, while calculations where an impenetrable trap is involved are naturally treated with a diffusion equation, standard trap problems are naturally treated within the SM. This difference is not relevant for particles without a bias in its diffusive motion, at least for the density calculations.

The organization of the paper is as follows. In the next section we briefly describe the model and discuss the differences between penetrable and impenetrable traps. In Sec. III we calculate the density as seen from a reference system attached to the (mobile) trap and discuss some symmetries. In the next section we make a similar analysis for the standard density, i. e. from the laboratory reference system, and calculate the number of particles absorbed by the trap. In Sec. V we present the results for the nearest neighbor distance. Finally we draw our conclusions.

\section{THE MODEL}

The model considers two species of particles, $A$ and $B$, both mobile and independent, and having a given reaction probability when they meet. Here we will study a one-dimensional trapping reaction in a system of $A$ particles uniformly distributed and a single trap $B$, with both species having a biased diffusive transport.

*Fellow of CNEA, Argentina; e-mail: sanchez@cab.cnea.gov.ar

${ }^{\dagger}$ http://www.cab.cnea.gov.ar/CAB/invbasica/FisEstad/estadis.htm 
The model equation for the evolution for $N(x, t)$, the density of the $A$ particles for a given realization of the trap trajectory $\epsilon(t)$, is the following

$$
\frac{\partial}{\partial t} N(x, t)=D_{A} \frac{\partial^{2}}{\partial x^{2}} N(x, t)-v_{A} \frac{\partial}{\partial x} N(x, t)-\gamma \delta(x-\epsilon(t)) N(x, t),
$$

where $\gamma$ is a constant measuring the reaction probability $\left(\gamma \rightarrow \infty\right.$ for perfect reactions), $D_{A, B}$ is the diffusivity and $v_{A, B}$ characterizes the bias of the $A$ or $B$ particles. In general we are interested in $n(x, t)=\langle N(x, t)\rangle$, the density averaged over realizations of $\epsilon(t)$ in the laboratory frame, or the similar (averaged) density $\tilde{n}(x, t)$ seen from a reference system attached to the trap.

As was pointed in Ref. [6] the SM equations given in Ref. [4] are valid using the propagators corresponding to the particle transport process. In our case we have for $A$ and $B$ particles

$$
\begin{aligned}
G(x, t) & =\frac{1}{\sqrt{4 \pi D_{A} t}} \exp \left[-\frac{\left(x-v_{A} t\right)^{2}}{4 D_{A} t}\right] \\
W(x, t) & =\frac{1}{\sqrt{4 \pi D_{B} t}} \exp \left[-\frac{\left(x-v_{B} t\right)^{2}}{4 D_{B} t}\right]
\end{aligned}
$$

respectively.

We will consider for a moment that the trap is fixed at the origin and the $A$ particles are not biased. Then Eq. (1) (in this case $N(x, t)=n(x, t)=\tilde{n}(x, t)$ ) becomes

$$
\frac{\partial}{\partial t} n(x, t)=D_{A} \frac{\partial^{2}}{\partial x^{2}} n(x, t)-\gamma \delta(x) n(x, t) .
$$

The resulting equation is equivalent to a diffusion equation except at the origin. It is not difficult to obtain the jump of the derivative in the density [11], which due to the symmetry results in the following boundary condition (for $x>0$ )

$$
\left.\frac{\partial}{\partial_{x}} n(x, t)\right|_{x=0}=\frac{\gamma}{2 D_{A}} n(0, t) .
$$

Hence the diffusion equation with this boundary condition is equivalent to Eq. (4). The same boundary condition could be obtained requiring that the diffusive current at the trap position (left minus right contributions) is proportional to the density, which is the usual way to obtain the so called radiative boundary condition for an imperfect trap. However, this is true due to the symmetry, which requires that the current at both sides of the trap are of equal magnitude. When coming to our bias problem (but with the trap immobile) the particles have a preferred direction of motion and the symmetry argument is not valid. On one hand, Eq. (1) models a penetrable trap but, although we can write an equation for the jump of the derivative we can not to write a equation for each lateral derivative separately. On the other hand, a current proportional to the density (from one particular direction) models an impenetrable trap. In this way we can see that each model is the natural way to treat each different situation. It is intuitive that for perfect trapping $(\gamma \rightarrow \infty)$ both models give the same density. A final remark is that the coefficient relating the diffusive current and the density at the trap position was taken as $\gamma$. This election make both models compatible. We must change $\gamma$ by $\gamma /\left(2 D_{A}\right)$ in the equations of Ref. [19] in order to make a direct comparison with results given in this work.

A detailed discussion for unbiased particles (and fixed trap) of penetrable and impenetrable traps was done by Taitelbaum [20]. Note that the analysis done here about impenetrable traps is only for a fixed trap, the case considered in Ref. [19]. The treatment for a mobile impenetrable trap is a non trivial generalization, and is out of the scope of this work.

\section{DENSITY IN THE TRAP FRAME}

In complete analogy with the case without bias 12] we change the reference system to one fixed to the trap. After doing the average it results that the density is similar to the case with a fixed trap, but changing $D_{A}$ by $D_{1}=D_{A}+D_{B}$ and $v_{A}$ by $v_{r}=v_{A}-v_{B}$. The resulting equation is solved by Laplace transform techniques, yielding after some calculations the expression

$$
\begin{aligned}
\frac{\tilde{n}(x, t)}{n_{0}}= & 1-\frac{1}{2} \frac{\mathrm{e}^{v_{r}(x-|x|) /\left(2 D_{1}\right)}}{1+v_{r} / \gamma} \operatorname{erfc}\left(\frac{|x|-v_{r} t}{\sqrt{4 D_{1} t}}\right)-\frac{1}{2} \frac{\mathrm{e}^{v_{r}(x+|x|) /\left(2 D_{1}\right)}}{1-v_{r} / \gamma} \operatorname{erfc}\left(\frac{|x|+v_{r} t}{\sqrt{4 D_{1} t}}\right) \\
& +\frac{1}{1-\left(v_{r} / \gamma\right)^{2}} \mathrm{e}^{\left(v_{r} x+\gamma|x|\right) /\left(2 D_{1}\right)+\left(\gamma^{2}-v_{r}^{2}\right) t /\left(4 D_{1}\right)} \operatorname{erfc}\left(\frac{|x|+\gamma t}{\sqrt{4 D_{1} t}}\right),
\end{aligned}
$$


valid for any value of the parameters except if $\left|v_{r}\right|=\gamma$. For $v_{r}=\gamma$ the expression is (expression for $v_{r}=-\gamma$ can be obtained performing a specular reflection)

$$
\begin{aligned}
\frac{\tilde{n}(x, t)}{n_{0}}= & 1-\frac{1}{4} \mathrm{e}^{v_{r}(x-|x|) /\left(2 D_{1}\right)} \operatorname{erfc}\left(\frac{|x|-v_{r} t}{\sqrt{4 D_{1} t}}\right)+\left[\frac{v_{r}}{4 D_{1}}\left(|x|+v_{r} t\right)+\frac{1}{4}\right] \\
& \times \mathrm{e}^{v_{r}(x+|x|) /\left(2 D_{1}\right)} \operatorname{erfc}\left(\frac{|x|+v_{r} t}{\sqrt{4 D_{1} t}}\right)-\frac{v_{r}}{2} \sqrt{\frac{t}{\pi D_{1}}} \mathrm{e}^{-\left(x-v_{r} t\right)^{2} /\left(4 D_{1} t\right)} .
\end{aligned}
$$

We can see that $\tilde{n}$ depends on the drift velocities only through the relative velocity $v_{r}$, and when both species have the same drift we recover the unbiased case. [13,21] In addition, a change in the sign of $v_{r}$ produce a specular reflection in the density profile. Hence, without loss of generality in the analysis we often set $v_{r}>0$. Note that in Eq. (6) the variables and parameters come only in three combinations: $x / \sqrt{D_{1} t}, v_{r} \sqrt{t / D_{1}}$ and $\gamma / v_{r}$.

Figure 1 shows a typical $\tilde{n}$ at different times and four sets of simulations corresponding to the same theoretical profile as all have the same $D_{1}$ and $v_{r}$. The agreement between the SM result and simulations is excellent.

We can observe that for $t \rightarrow \infty$ the density in the trap position approaches

$$
\frac{\tilde{n}(0, t)}{n_{0}} \sim \frac{\left|v_{r}\right|}{\gamma+\left|v_{r}\right|},
$$

which is a positive value (for imperfect trapping), in contrast with the unbiased case $\left(v_{r}=0\right)$ where the particle density asymptotically reaches zero at the trap position. [13]21] Besides, for $v_{r}>0$ the density reaches a stationary profile $\left(\tilde{n}(x, t) / n_{0} \sim 1-\exp \left(v_{r} x / D_{1}\right) /\left(1+v_{r} / \gamma\right)\right)$ in the left semi-axis, i. e. where the relative drift is towards the trap, while any point to the right of the trap reaches asymptotically the value given by Eq. (8). These aspects can be seen in Fig. 1.

\section{DENSITY IN THE LABORATORY FRAME}

The Laplace-Fourier transform of the density in the laboratory frame [4] is given by

$$
\begin{aligned}
\frac{n(k, s)}{n_{0}}= & \frac{2 \pi \delta(k)}{s}-\frac{\gamma}{\left(s+D_{A} k^{2}+i k v_{A}\right)\left(s+D_{B} k^{2}+i k v_{B}\right)} \\
& \times \frac{1}{1+\gamma\left[4 D_{1} s+v_{r}^{2}+4 D_{A} D_{B} k^{2}+4 i k\left(v_{A} D_{B}+v_{B} D_{A}\right)\right]^{-1 / 2}} .
\end{aligned}
$$

Here we can see that Eq. (9) does not change if we interchange $D_{A}$ by $D_{B}$ and $v_{A}$ by $v_{B}$ simultaneously. This fact shows that the trap and the particles play exactly the same role in the density. However, this characteristic is not valid for any magnitude, as is known from the first neighbor distance in the unbiased problem [12]. The equivalence between both particle species just mentioned lead to two interesting consequences that we describe in the following.

The first consequence is a symmetric density if we set $D_{A}=D_{B}=D$ and $v_{A}=-v_{B}=v$. This can be verified considering that from Eq. (9) results $n(k, s)=n(-k, s)$. To obtain the general expression in the $x$ and $t$ variables is very difficult and we present the simple result for perfect absorption

$$
\begin{aligned}
\frac{n(x, t)}{n_{0}}= & \frac{1}{2}+\frac{1}{2} \operatorname{erf}\left(\frac{x+v t}{\sqrt{4 D t}}\right) \operatorname{erf}\left(\frac{x-v t}{\sqrt{4 D t}}\right) \\
& +\frac{2}{v} \sqrt{\frac{D}{\pi t}}\left[\mathrm{e}^{-(x-v t)^{2} /(4 D t)} \operatorname{erf}\left(\frac{x+v t}{\sqrt{4 D t}}\right)-\mathrm{e}^{-(x+v t)^{2} /(4 D t)} \operatorname{erf}\left(\frac{x-v t}{\sqrt{4 D t}}\right)\right] .
\end{aligned}
$$

This expression has only two variables (any parameter) that are $x / \sqrt{4 D t}$ and $v \sqrt{t /(4 D)}$. We note that this expression approaches asymptotically zero at the origin, in contrast with the unbiased case where the density reaches a finite value. [13,21] In Fig. 2 we can see an example of symmetric profiles at different times. The agreement between theory and simulation is good, although there is a systematic difference. However, note that the symmetry is still present in simulations.

The second consequence is that setting $D_{B}=v_{B}=0$ (fixed trap) the density expression is the same as setting $D_{A}=v_{A}=0$ (fixed particles) exchanging the particle subindexes. Moreover, since for the fixed trap we have the trivial equality $\tilde{n}(x, t)=n(x, t)$, and $\tilde{n}(x, t)$ for $D_{B}=v_{B}=0$ is equal to $\tilde{n}(-x, t)$ for $D_{A}=v_{A}=0$, we have $\tilde{n}(-x, t)=n(x, t)$ for fixed particles, which is not evident. A similar property was found in the unbiased case [13]. 
In Fig. 3 we show simulations of $\tilde{n}(x, t)$ and $n(x, t)$ for fixed particles, $n(x, t)$ for the fixed trap and the SM result corresponding to all of them. We can see an excellent agreement.

By setting $k=0$ in the second term of Eq. (9), the total number of absorbed particles in the Laplace domain can be obtained. Performing the inverse transform we obtain

$$
\begin{aligned}
\frac{N_{A B S}(t)}{n_{0}}= & \frac{2 \gamma^{2} \sqrt{D_{1} t}}{\sqrt{\pi}\left(\gamma^{2}-v_{r}^{2}\right)} \mathrm{e}^{-v_{r}^{2} t /\left(4 D_{1}\right)}+\frac{4 D_{1} \gamma^{3}}{\left(\gamma^{2}-v_{r}^{2}\right)^{2}} \mathrm{e}^{\left(\gamma^{2}-v_{r}^{2}\right) t /\left(4 D_{1}\right)} \operatorname{erfc}\left(\gamma \sqrt{\frac{t}{4 D_{1}}}\right) \\
& +\frac{\gamma\left|v_{r}\right| t}{\gamma+\left|v_{r}\right|}+\frac{2 D_{1}}{\left|v_{r}\right|} \frac{\gamma^{2}}{\left(\gamma+\left|v_{r}\right|\right)^{2}} \\
& -\left[\frac{2 D_{1} \gamma^{2}}{\left|v_{r}\right|} \frac{\gamma^{2}+v_{r}^{2}}{\left(\gamma^{2}-v_{r}^{2}\right)^{2}}+\frac{\gamma^{2}\left|v_{r}\right| t}{\gamma^{2}-v_{r}^{2}}\right] \operatorname{erfc}\left(\left|v_{r}\right| \sqrt{\frac{t}{4 D_{1}}}\right) .
\end{aligned}
$$

The limit cases $\gamma \rightarrow \infty$ and $\gamma=\left|v_{r}\right|$, not included for the sake of brevity, can be extracted from Eq. (11) taking the corresponding limit. In Fig. 4 we show simulations of $N_{A B S}$ for the same sets of parameters than in Fig. 1. Simulation data coming from all sets are rather coincident (almost indistinguishable in the figure) and in excellent agreement with the SM prediction. If $v_{A}=v_{B}$ Eq. (11) coincides with the case without bias, where asymptotically $N_{A B S} \propto t^{1 / 2}[12]$. However for $v_{r}=v_{A}-v_{B} \neq 0$ the asymptotic behavior results

$$
\frac{N_{A B S}(t)}{n_{0}} \sim \frac{\gamma\left|v_{r}\right|}{\left|v_{r}\right|+\gamma} t
$$

This change in the behavior can be understood from Eq. (8), since the density at the trap position reaches a positive value. Hence, the absorption rate $\gamma \tilde{n}(0, t)$ becomes constant. The same arguments are applicable to an impenetrable trap that has the same qualitative dependence (although the factor is different because $\tilde{n}(0, t)$ is different).

It is possible to factorize Eq. (9) (after performing the inverse Laplace transform) in $\exp \left(-i k v_{B} t\right)$ and an expression where $v_{A}$ and $v_{B}$ arise only in the $v_{r}$ combination. This implies that in the $x$ domain the density has the form $n(x, t)=n^{*}\left(x-v_{B} t\right)$, where $n^{*}$ depends on $v_{r}$ but neither on $v_{A}$ nor $v_{B}$ separately. Due to the identical role played in the density expression by both particle species, we can do a similar factorization involving $\exp \left(-i k v_{A} t\right)$.

In Eq. (9) we take the limit $\gamma \rightarrow \infty$ in order to obtain the mathematically simple expression for perfect reaction resulting, after performing the inverse Laplace transform, in

$$
\begin{aligned}
\frac{n(k, t)}{n_{0}}= & 2 \pi \delta(k)-\frac{2}{k^{2}\left(D_{B}-D_{A}\right)-i k v_{r}}\left\{\left(i k D_{A}-\frac{v_{r}}{2}\right) \mathrm{e}^{-\left(D_{A} k^{2}+i k v_{A}\right) t} \operatorname{erf}\left[\left(i k D_{A}-\frac{v_{r}}{2}\right) \sqrt{\frac{t}{D_{1}}}\right]\right. \\
& \left.-\left(i k D_{B}+\frac{v_{r}}{2}\right) \mathrm{e}^{-\left(D_{B} k^{2}+i k v_{B}\right) t} \operatorname{erf}\left[\left(i k D_{B}+\frac{v_{r}}{2}\right) \sqrt{\frac{t}{D_{1}}}\right]\right\} .
\end{aligned}
$$

Numerically performing the inverse Fourier transform we obtain the density in the $x$ domain. In Fig. 5 we show numerical integrations at different times together with the respective simulations. The agreement between both results is apparent.

\section{NEAREST NEIGHBOR-DISTANCE}

As was stated in Ref. 12], we can use the expression for the PDF calculated in a similar way as for fixed particles as an approximation to the problem of both mobile species. This approximation is better for a small $D_{B} / D_{A}$ ratio and for short times. We note that we can consider biased particles as having a deterministic drift velocity plus an unbiased diffusion. Hence, following Ref. 12] the PDF only depends on $v_{r}$ (at variance with diffusivities where the dependence is not trivial). To calculate the PDF of the (one sided) nearest neighbor distance we first compute the intermediate quantity $Q(x, t)=\exp \left(-\int_{0}^{x} \tilde{n}(u, t) d u\right)$, and then the PDF is $p(x, t)=-\partial_{x} Q(x, t)=\tilde{n}(x, t) Q(x, t)$. 2224 The integral involved in $Q$ can be done analytically, yielding a rather complicated expression.

In Fig. 6 we show a plot of $p$ corresponding to the right neighbor and compare it with simulations. We can see a good agreement. The most noticeable difference arises near the origin. However, the mean distance $\langle x\rangle=\int_{0}^{\infty} p(x, t) x d x=$ $\int_{0}^{\infty} Q(x, t) d x$ shows the expected systematic differences due to the approximation. This is apparent in Fig. 7 where we show $\langle x\rangle$ coming from a numerical integration. Note that squares and diamonds are almost coincident in Fig. 7 (because they have the same parameters except the drift velocities but with the same $v_{r}$ ). In addition we make a similar calculation and simulations for the left neighbor. In this case the mean distance reaches a stationary value that can be calculated within the SM and is 


$$
\langle x\rangle=\frac{D_{1}}{v_{r}}\left[\left(1+\frac{v_{r}}{\gamma}\right) \frac{v_{r}}{n_{0} D_{1}}\right]^{n_{0} D_{1} / v_{r}} \exp \left(\frac{n_{0} D_{1}}{v_{r}} \frac{\gamma}{\gamma+v_{r}}\right) \bar{\gamma}\left(\frac{n_{0} D_{1}}{v_{r}}, \frac{n_{0} D_{1}}{v_{r}} \frac{\gamma}{\gamma+v_{r}}\right)
$$

where $\bar{\gamma}(a, z)$ is the incomplete gamma function 25 (we have the over bar to avoid confusions with the absorption constant). We can see in the figure that this quantity does not have a good agreement with simulations. This is due to the fact that the model is a continuous one and can not describe well the situation very near the trap, where the discreteness of the problem is relevant. This phenomenon is the same as in Fig. 6 (small $x$ ), but here it is more noticeable because almost all particles in the simulation are in the same place of the trap or in the nearest neighbor site.

Simulations were performed on a lattice of $L$ sites, with periodic boundary conditions. We have used the same algorithm described in [12], with a small change to allow for the drift of the particles. The connections between the simulation and SM parameters are $D=\omega_{j} \Delta x^{2} / 2, v=(2 q-1) \omega_{j} \Delta x$ and $\gamma=\omega_{r} \Delta x$; where $\Delta x$ is the jump length, $\omega_{j, r}$ are the jump and reaction frequencies respectively, and $q$ is the probability of a given jump to the right $(q=0.5$ corresponds to an unbiased particle). All show simulations are the average of 10000 realizations over a 200 site lattice and with an initial density $n_{0}=1$.

\section{CONCLUSIONS}

We have studied a trapping reaction $A+B \rightarrow B$ with a single penetrable trap $B$ in a one dimensional lattice, where both species are mobile and perform a biased diffusion.

We have seen that the natural tool to perform analytical calculations is the stochastic model (SM) in contrast with a diffusion equation that is useful for calculations involving an impenetrable trap. We have calculated the expressions of $\tilde{n}(x, t)$, the distribution of particles in the trap frame, and $n(x, t)$, the usual density in the laboratory frame.

Analogously to the unbiased case, $\tilde{n}(x, t)$ can be obtained from the fixed trap case doing the exchange $D_{A} \rightarrow D_{1}=$ $D_{A}+D_{B}$ and $v_{A} \rightarrow v_{r}=v_{A}-v_{B}$ in the corresponding expression. In the general expression we note that $\tilde{n}(0, t)$ for imperfect reactions reaches a non zero value at long time. This results in a linear asymptotic number of absorbed particles, in contrast with the $t^{1 / 2}$ behavior of the unbiased problem.

The expression for $n(x, t)$ has the following symmetry: it is invariant by exchange of diffusivities and velocities simultaneously, i. e. $D_{A} \rightarrow D_{B}, D_{B} \rightarrow D_{A}, v_{A} \rightarrow v_{B}, v_{B} \rightarrow v_{A}$. This is valid both for perfect and imperfect reactions. Due to this characteristic there is a combination of parameters, namely $D_{A}=D_{B}$ and $v_{A}=-v_{B}$, which results in a symmetric density. Another consequence is that $n(x, t)=\tilde{n}(-x, t)$ for fixed $A$ particles, a property found first in the unbiased case. We note that in the symmetric case for perfect reaction the particles are asymptotically extinguished, in contrast with the unbiased case where the density reaches a positive value. An explanation of this fact is that the trap in average goes to the right and particles located to its right can not pass through it to reach the origin (because it is a perfect trap). In addition particles situated to the left have in average a velocity to the left. Hence the origin results asymptotically free of particles yielding the observed null density.

A comparison of the density profile with that obtained for an impenetrable trap, when the trap is fixed (where we have available results from Ref. [19]), shows that both expressions are similar if the absorption rate is high. In fact, for perfect absorption both densities become identical. On the contrary, for low absorption an impenetrable trap implies that the untrapped particles are accumulated (to the left if $v_{A}>0$ ) while they follow their way to the right for a penetrable trap. In spite of this, both problems reach a stationary profile to the left of the trap (for $v_{A}>0$ ).

We have studied the nearest neighbor distance in the case of $D_{B}=0$, and used an approximation valid for small $D_{B}$. We can see that the mean distance, after a short transient, approaches a constant to the left of the trap, while it grows to the right of the trap.

Simulations are in excellent agreement with the model. As was mentioned in the introduction, this is an expected result since the model has been solved exactly. Finally, we want to remark that while in unbiased particle problems the SM is a convenient alternative to other technics, in problems of biased particles it becomes the natural way to obtain analytical results.

\section{ACKNOWLEDGMENTS}

Financial support from CONICET (Project PIP-4953/96) and ANPCyT (Project 03-00000-00988), Argentina is acknowledged. The author wants to thank H. S. Wio for the careful revision of the manuscript. 
[1] S. A. Rice., Diffusion-Limited Reactions, Elsevier, Amsterdam, 1985.

[2] A. D. Galanin, Thermal Reactor Theory, second edition, Pergamon Press, New York, 1960. M. M. R. Williamas, Random Processes in Nuclear Reactors, Pergamon Press, Oxford, 1974.

[3] I. Martinez and M. A. Rodriguez, Ann. Nuc. Energy 12, 113 (1985).

[4] M. A. Rodriguez, G. Abramson, H. S. Wio and A. Bru, Phys. Rev. E 48, 829 (1993).

[5] G. Abramson, Ph.D. thesis, Instituto Balseiro, Universidad Nacional de Cuyo, Argentina, 1995; A. Bru, Ph.D. thesis, Universidad Complutense de Madrid, Spain, 1995.

[6] G. Abramson, A. Bru Espino, M. A. Rodriguez and H. S. Wio, Phys. Rev. E 50, 4319 (1994).

[7] H. S. Wio, G. Abramson, M. A. Rodriguez and A. Bru, Chaos, Solit. \& Fract. 6, 575 (1995).

[8] G. Abramson and H. S. Wio, Chaos, Solit. \& Fract. 6, 1 (1995).

[9] A. D. Sánchez, E. M. Nicola and H. S. Wio, Phys. Rev. Lett. 78, 2244 (1997).

[10] G. Abramson and H. S. Wio, Phys. Rev. E 53, 2265 (1996).

[11] A. D. Sánchez and H. S. Wio, Physica A 237, 452 (1997).

[12] A. D. Sánchez, M. A. Rodriguez and H. S. Wio, Phys. Rev. E 57, 6390 (1998).

[13] A. D. Sánchez, Phys. Rev. E 59, 5021 (1999).

[14] A. D. Sánchez, S. Bouzat and H. S. Wio, Phys. Rev. E 60, 2677 (1999).

[15] J. R. Philip, J. H. Knight, and R. T. Waechter, Water Resour. Res. 25, 16 (1989).

[16] J. H. Knight, J. R. Philip, and R. T. Waechter, Water Resour. Res. 25, 29 (1989).

[17] P. J. Schultz and K. G. Lynn, Rev. Mod. Phys. 60, 701 (1988).

[18] H. Nakajima and H. B. Huntington, J. Phys. Chem. Solids 42, 171 (1981).

[19] C. A. Condat, G. Sibona and C. E. Budde, Phys. Rev. E 51, 2839 (1995).

[20] H. Taitelbaum, Physica A 190, 295 (1992).

[21] Z. Koza and H. Taitelbaum, Phys. Rev. E 57, 237 (1998)

[22] G. H. Weiss, R. Kopelman and S. Havlin, Phys. Rev. A 39, 466 (1989).

[23] S. Redner and D. ben-Avraham, J. Phys. A 23, L1169 (1990).

[24] H. Taitelbaum, R. Kopelman, G. H. Weiss and S. Havlin, Phys. Rev. A 41, 3116 (1990).

[25] M. Abramowitz y I. A. Stegun, Handbook of mathematical functions, Dober, New York 1972.

FIG. 1. Density of $A$ particles in the reference frame of the trap at different times. Simulations are performed with different parameter sets indicated in the figure. All of them have $D_{1}=0.2$ and $v_{r}=0.16$. The reaction is imperfect with $\gamma=1$.

FIG. 2. Density of $A$ particles in the symmetric case, as seen from the laboratory coordinate system, for a perfect reaction. The simulation parameters are: $D_{A}=D_{B}=0.1$ and $v_{A}=-v_{B}=0.08$.

FIG. 3. Simulation for the density of fixed $A$ particles in the reference frame of the trap, from the laboratory coordinate system, and for the fixed trap. The parameters are: $D_{A}$ or $D_{B}=0.1,\left|v_{A}\right|$ or $\left|v_{B}\right|=0.08$ and $\gamma=1$.

FIG. 4. Number of absorbed particles. The parameters are the same as those of the Fig. 1

FIG. 5. Density of $A$ particles, as seen from the laboratory coordinate system, for a perfect reaction. The simulation parameters are: $D_{A}=0.2, D_{B}=0.1, v_{A}=0.16$ and $v_{B}=-0.08$.

FIG. 6. PDF of the nearest neighbor distance to the trap at $t=200$. The parameters, indicated in the figure, are the same as in Fig. 1.

FIG. 7. Mean distance to the nearest neighbor to the trap. The parameters, indicated in the figure, are the same as in Fig. 1. 
Fig. 1 Sánchez

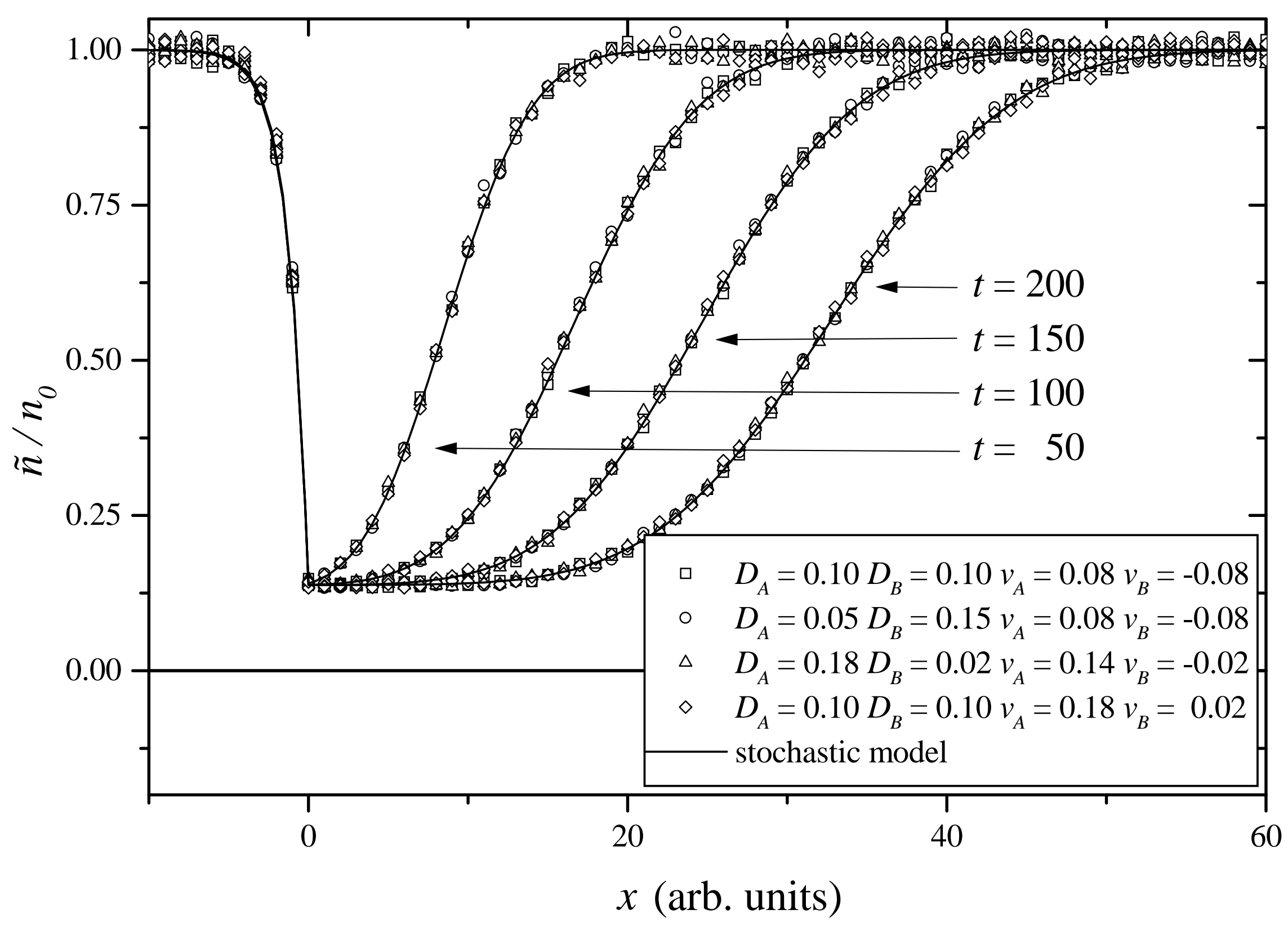


Fig. 2 Sánchez

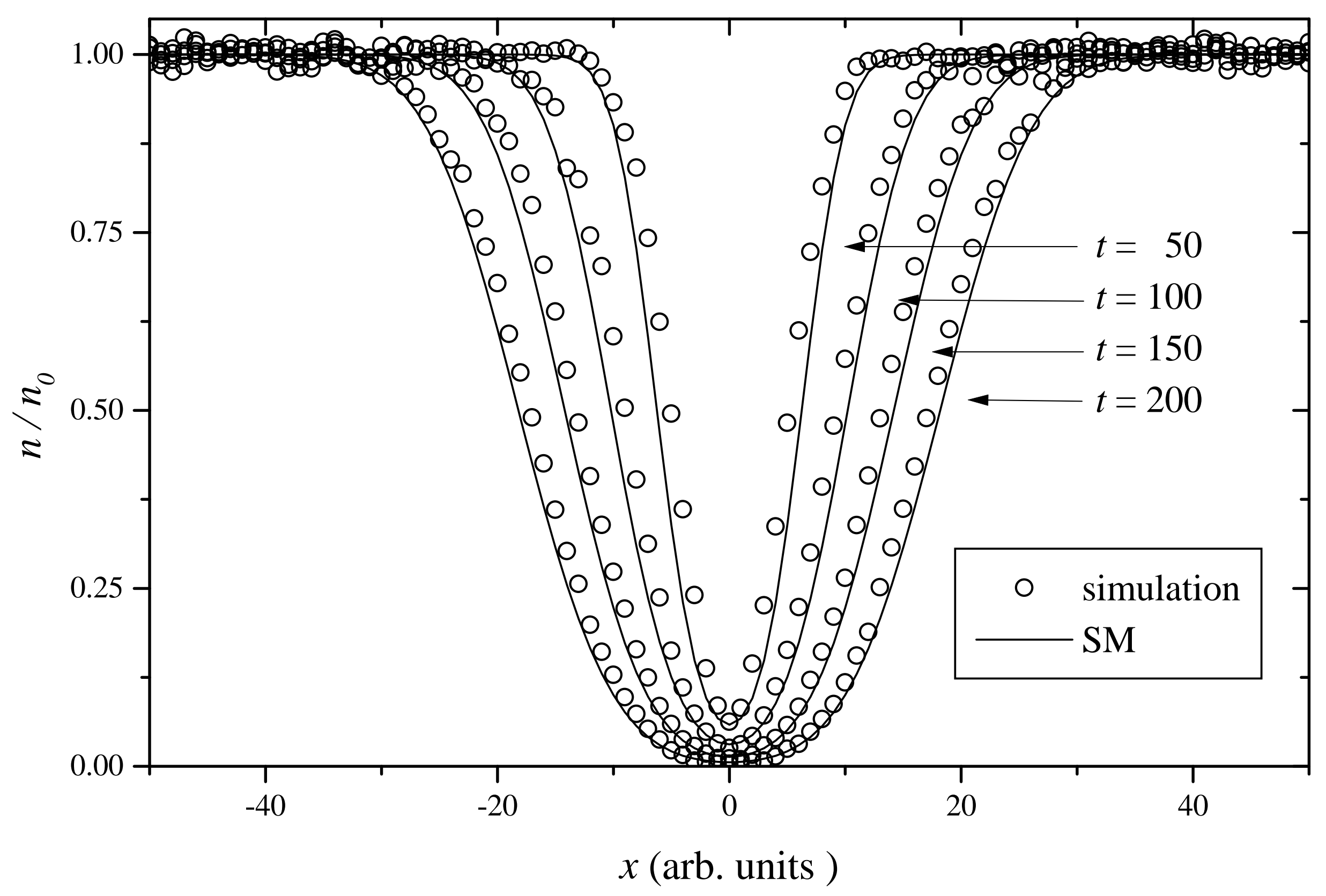


Fig. 3 Sánchez

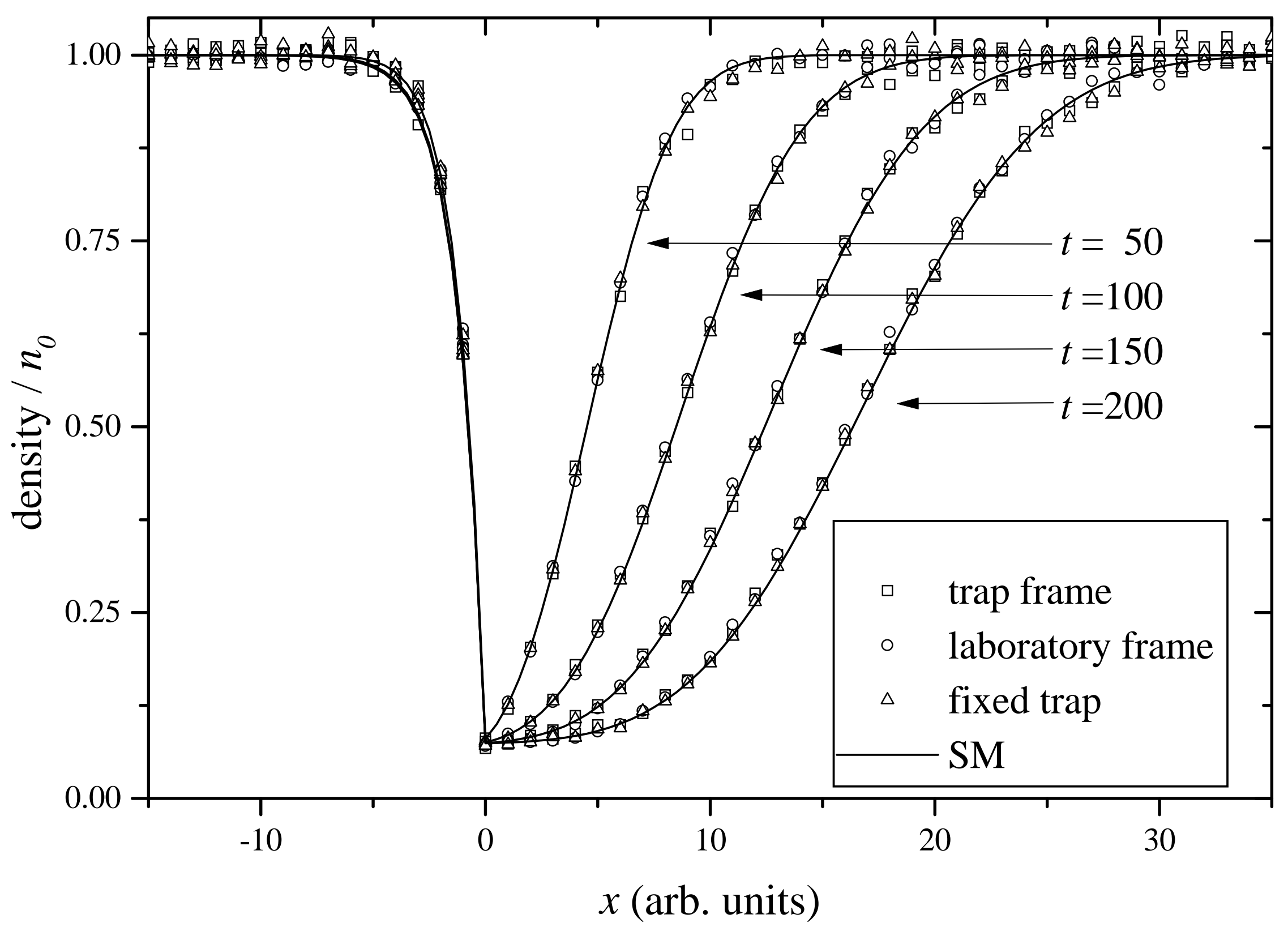


Fig. 4 Sánchez

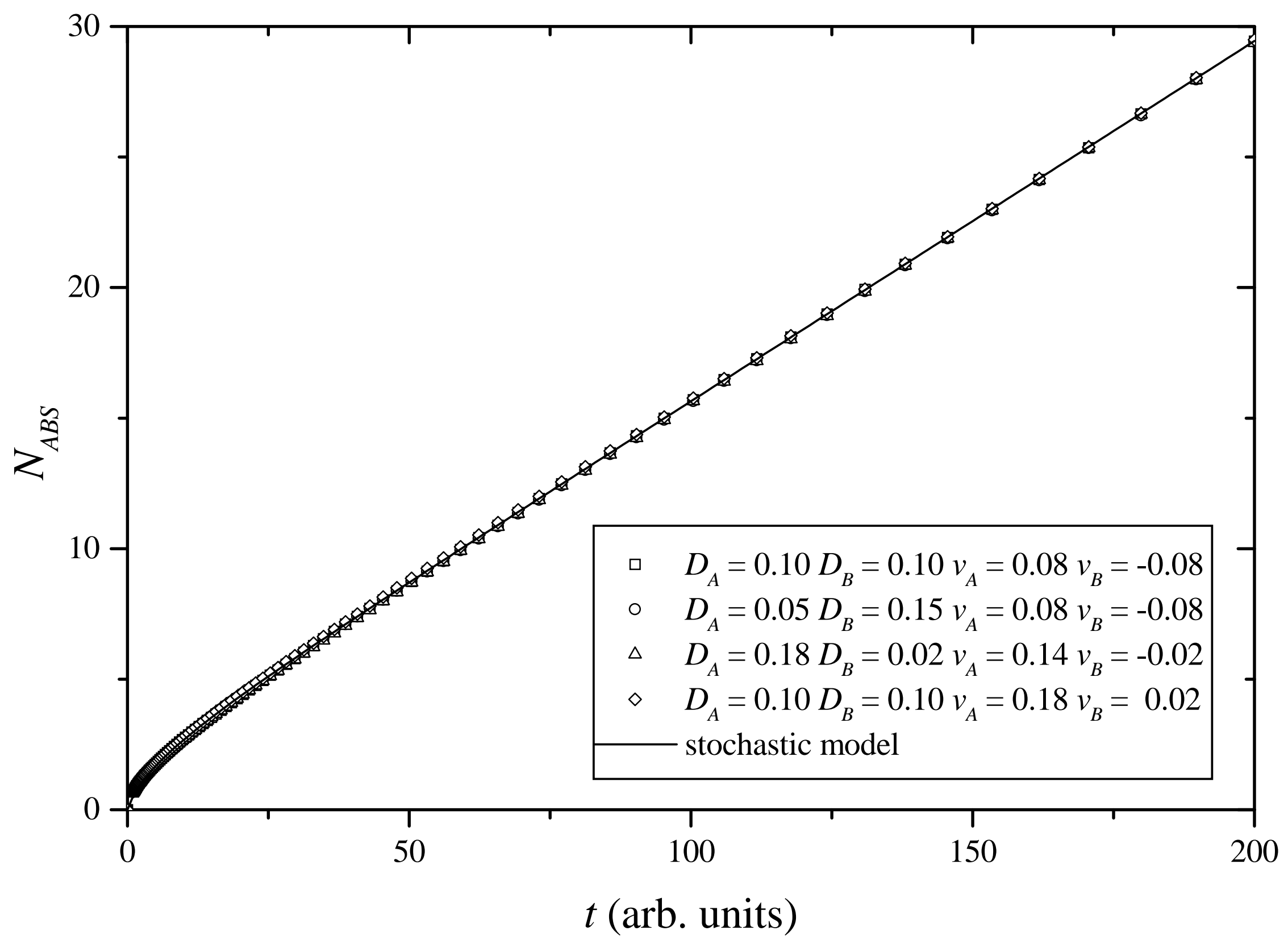


Fig. 5 Sánchez

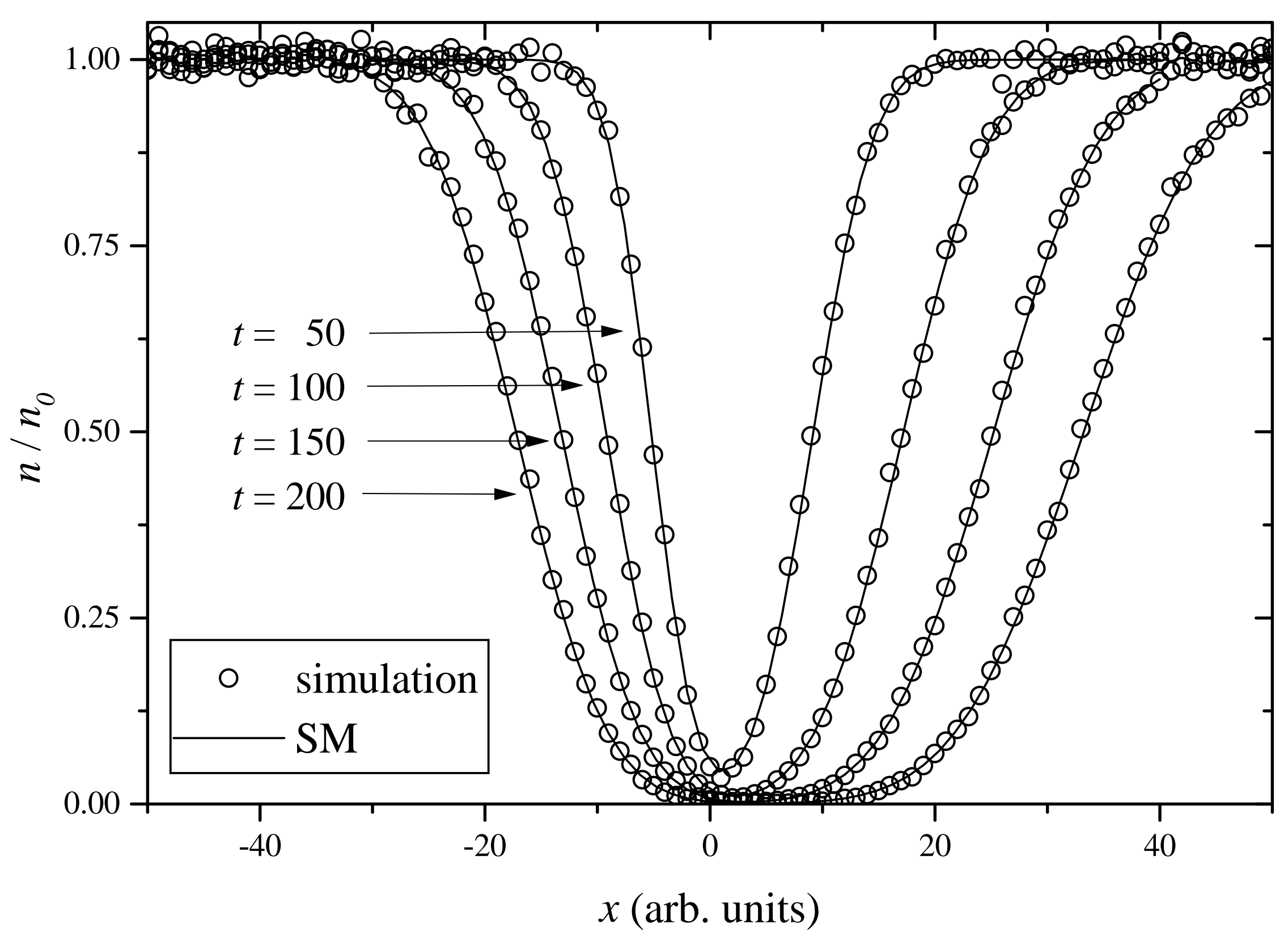


Fig. 6 Sánchez

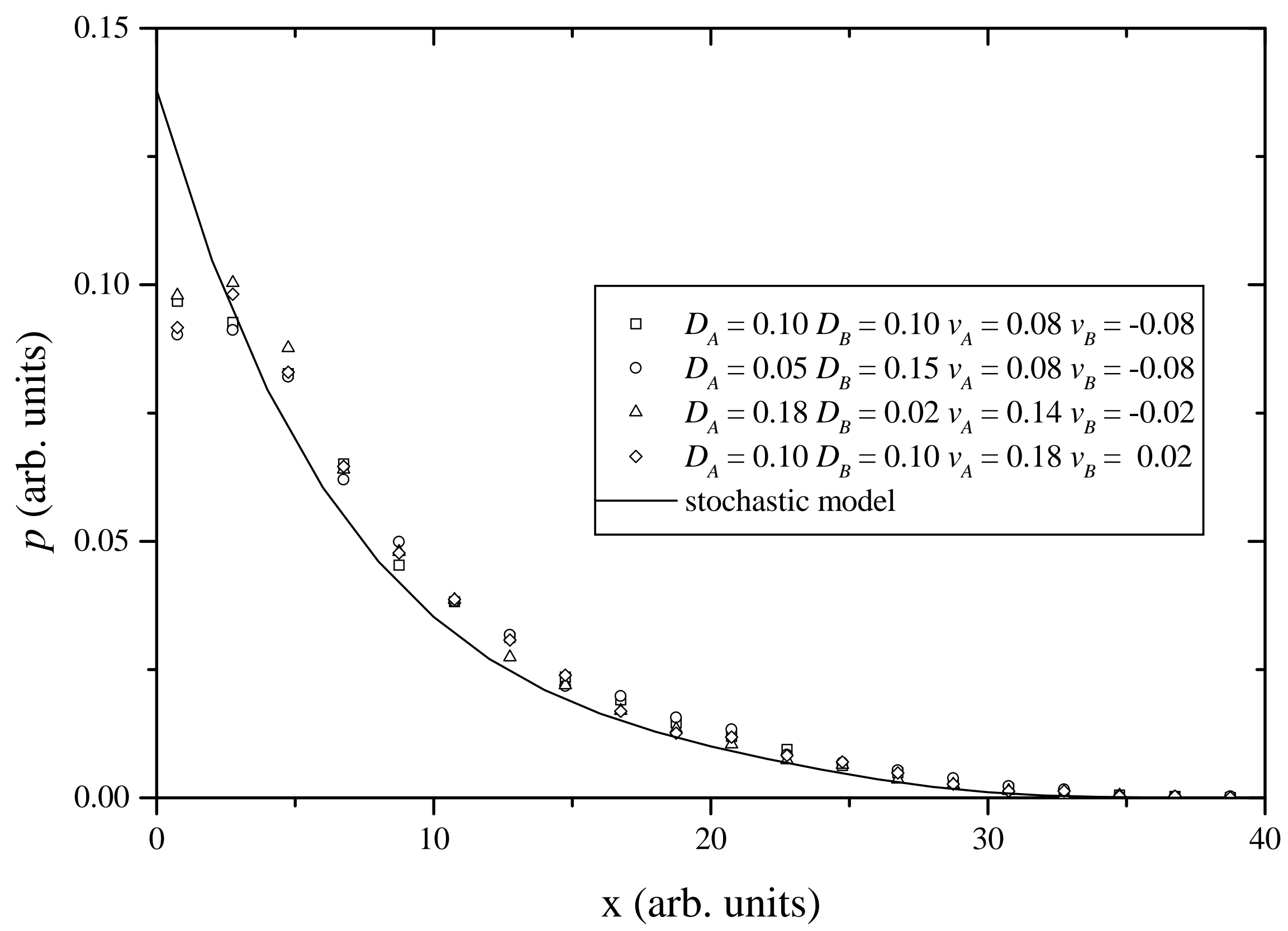


Fig. 7 Sánchez

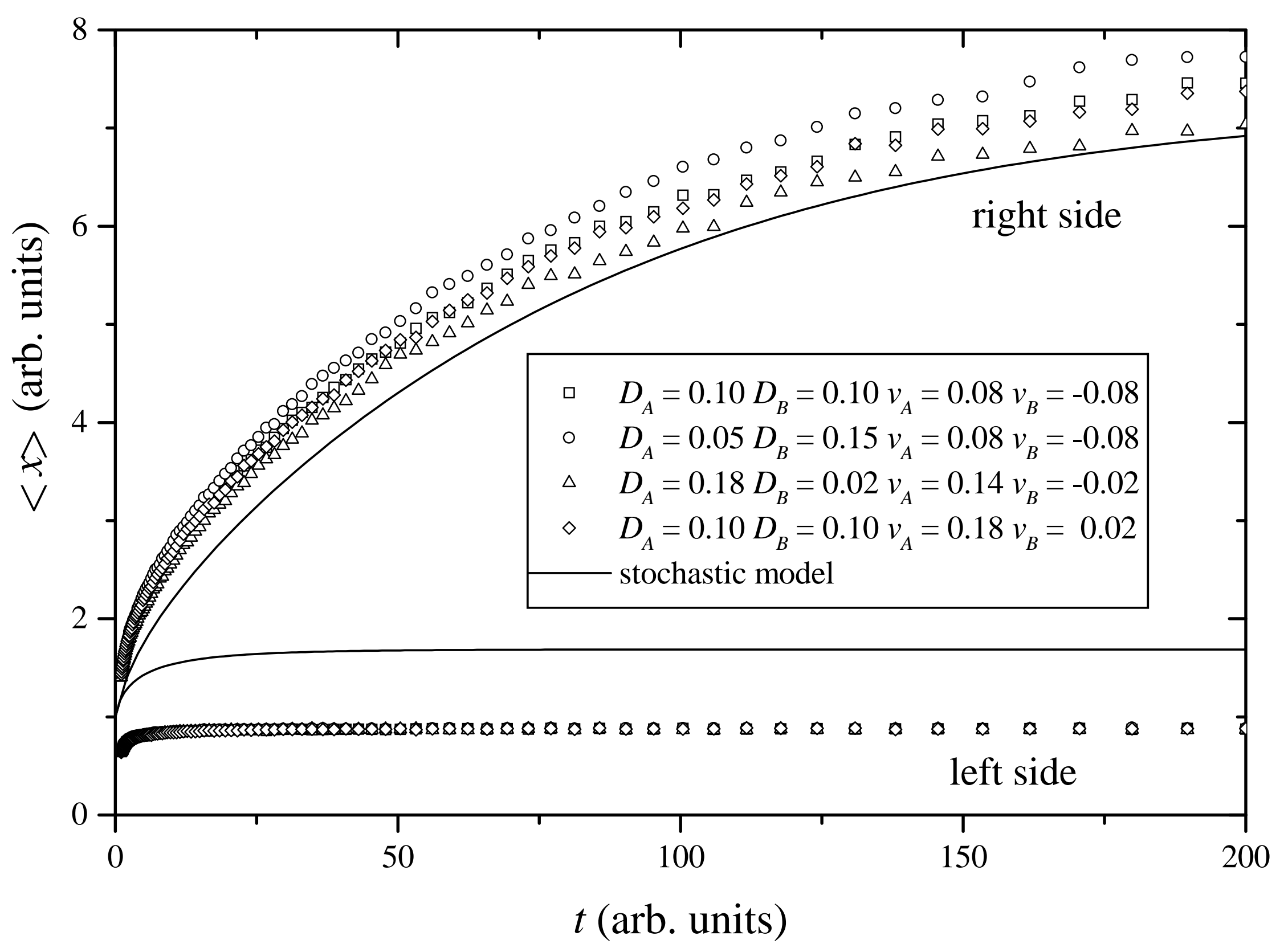

